\title{
Noise Correlation-Aided Iterative Decoding for Magnetic Recording Channels
}

\author{
Pisit Vanichchanunt ${ }^{1}$, Kampol Woradit ${ }^{1}$, Suvit Nakpeerayuth ${ }^{1}$, Lunchakorn Wuttisittikulkij ${ }^{1}$, \\ and Lajos Hanzo ${ }^{2}$ \\ ${ }^{1}$ Department of Electrical Engineering, Chulalongkorn University, Bangkok, Thailand \\ ${ }^{2}$ School of Electronics and Computer Science, University of Southampton, SO17 1BJ, UK \\ Email: pisit.v@student.chula.ac.th, suvit@ee.eng.chula.ac.th, wlunchak@chula.ac.th, \\ and lha ecs.soton.ac.uk
}

\begin{abstract}
In this paper, three noise correlation-aided iterative decoding schemes are proposed for magnetic recording channels, where the correlation is imposed by the equalizer's spectral shaping effect. The first approach exploits the noise' correlation in the form of linear prediction-aided detection by increasing the number of detector trellis states invoked by the Bahl, Cocke, Jelinek, and Raviv (BCJR) detection algorithm. In the second approach, we have extended the first technique by employing both previous and future correlated noise samples in order to attain noise estimates. In order to achieve this objective, the classic BCJR algorithm has to be modified for the sake of additionally exploiting future noise samples. The third approach is designed for reducing the decoding complexity by applying the Viterbi Algorithm (VA) to assist the detector in finding the encoded sequences associated with the survivor paths in the detector's trellis, without increasing the number of trellis states. We will demonstrate that for the classic PR4-equalized Lorentzian channel, the proposed schemes are capable of offering a performance gain in the range of 1.1-3.7 dB over that of a benchmark turbo decoding system at the BER of $10^{-5}$ and at a recording density of 2.86 .
\end{abstract}

Keywords-magnetic recording, noise prediction, turbo codes.

\section{INTRODUCTION}

In magnetic recording devices, data is written and read back and forth between the read/write head(s) and the magnetic storage medium. This process can be characterized by the widely accepted model of the saturation-based magnetic recording channel, namely by the Lorentzian channel model [1]. The step response of this channel is assumed to be the Lorentzian pulse [1]. Since the data pulse shape is spread by the long-memory channel, Intersymbol Interference (ISI) is inevitably encountered, especially at a high recording density [2]. Although maximum-likelihood sequence estimation (MLSE) based equalization can be applied for mitigating the ISI, it may have an excessive complexity.

Kobayashi and Tang [2] suggested that partial-response (PR) signaling [3] has the ability of coping with the ISI of magnetic recording channels by equalizing the ISI-contaminated signal. Then, the MLSE-based Viterbi algorithm (VA) can be used for detecting the data sequence at an acceptable complexity [4]-[5], and this process is often referred to as partial-response signaling using maximum-likelihood sequence estimation (PRML).

In [6], Thapar and Partel extended the contribution of [4] and [5] by proposing a polynomial-based class of partial response signaling for magnetic recording channels, where the signaling waveform was $g_{P R}(D)=(1-D)(1+D)^{n}, n=0,1,2, \ldots$, and $n$ has to be increased for the sake of increasing the achievable recording

This work was supported by the Royal Golden Jubilee Ph.D. Program of the Thailand Research Fund and the Cooperation Project between the Department of Electrical Engineering and Private Sector for Research and Development. density, which may be attained upon increasing the pulse-shaping equalization and decoding complexity. The factor $(1-D)$ is used to account for the spectral null of recording channels at DC whereas the factor $(1+D)^{n}$ matches the high-frequency attenuation of the channel resulting from losses due to the head-tomedium spacing and owing to the finite recording transition width [24]. For $n=0$, the channel $g_{P R}(D)=(1-D)$ is referred to as the dicode partial response channel. The channel associated with $n=1$ is known as the class-4 partial response (PR4) channel. Finally, the channel using $n=2$ is termed as the extended class- 4 partial response (EPR4) channel, while for $n=3$ we refer to the extended EPR4 (E²PR4) channel [7].

As a benefit of their capability of coping with the ISI, PRML techniques have been predominant in detectors designed for magnetic recording channels since the 1990s [15]. At high recording densities, powerful equalization is required which imposes a high noise correlation at the input of the detector, hence potentially resulting in a degradation of the detector's performance. In order to improve the VA's attainable performance, Chevillat et al. [8] exploited the equalizer-induced noise correlation by using a linear predictor scheme for whitening the noise spectrum at the input of the VA-aided detector. Four years later, Eleftheriou and Hirt [9] proposed a detection scheme, which embedded a noise prediction and whitening process into the branch metric computation of a VA-based detector in order to improve the achievable detection performance in the context of the PR4 and EPR4 channels. In [10], Coker et al. interpreted the combination of the PR4 channel and the linear predictor filter polynomial as a generalization of partial response signaling (see introduction of [11]), which has the capability of shaping the ISIcontaminated signal, while also whitening the noise of magnetic recording channels.

Iterative decoding [12]-[13] has found wide-ranging applications over the past decade, including magnetic recording. Furthermore, advanced iterative decoding techniques operating both with [14]-[16] and without noise prediction [17]-[20] have also been devised. More specifically, in [14] the signal-dependent noise induced by the signaling-pulse position jitter imposed by tape vow and flatter, was modeled and predicted for the sake of improving the performance of low-density parity check codes (LDPC). In [15] both hard and tentative soft decisions were employed for improving the performance of an iteratively decoded system using a recursive systematic convolutional encoder. The $a$ posteriori probability ( $\mathrm{APP}$ ) of the coded bits was calculated and used for estimating the average values of both the channel output and noise for the sake of improving the achievable performance. Although impressive performance results were attained, the number of iterations required was as high as 16-20. In [16] iterative decoding was invoked in the context of an AutoRegressive (AR) channel model.

Against this background, in this paper, a turbo decoding scheme designed for equalized Lorentzian channels contaminated by colored noise is proposed. Hence, we first modify the classic APP detector so that it becomes capable of exploiting the correlated nature of the noise, which was colored by the overlap- 


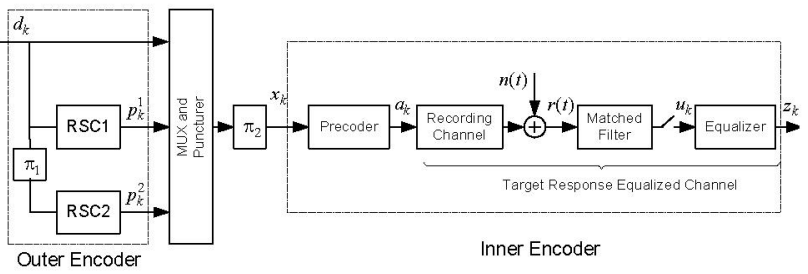

Fig. 1. Encoding scheme and the equalized channel

pulse-filtering effect of the matched filter and by the spectral shaping effect of the equalizer. It is then shown that the BCJR algorithm [21] is capable of exploiting the correlation of the noise, manifesting itself in terms of predictability upon employing linear prediction. However, this is achieved at the cost of exponentially increasing the number of the detector trellis states as a function of the linear predictor order. This approach is reminiscent of the iterative Multiple Symbol Differential Detection (MSDD) schemes of [22], [23]. Based on [22] and [23], the question arises, whether the detector is capable of exploiting the colored noise' correlation more intelligently, namely by using both forward and backward prediction in order to further improve its achievable performance. We answer this question positively by proposing a novel approach, which is capable of improving the performance of magnetic recording systems at high recording densities. Similar to the previously mentioned linear predictive technique, the proposed approach results in an increased number of trellis states, when invoking both previous and future noise samples in the linear estimation process. We will demonstrate that given the PR4 partial response channel, the proposed approach is capable of outperforming its counterpart using only single-sided linear prediction when aiming at a high recording density.

The rest of this paper is organized as follows. The encoding scheme is outlined in Section II. The equalized Lorentzian channel model using the classic Minimum Mean Square Error (MMSE) equalizer for shaping the spectrum of the channel output is reviewed in Section III. The decoding schemes used are described in Section IV, while our performance results are detailed in Section V. Finally, our conclusions are offered in Section VI.

\section{ENCODING SYSTEM}

The encoding system shown in Fig. 1 comprises a parallel concatenated convolutional encoder, a MUX/puncturing block, a channel interleaver $\pi_{2}$, and a precoder [18]. The data bit sequence $d_{1}, d_{2}, \ldots, d_{N}$ denoted as $\underline{d}_{1}^{N}$ having a length of $N$ is encoded by the Recursive Systematic Convolutional encoders RSC1 and $\mathrm{RSC} 2$, where RSC2 is fed by the turbo interleaver $\pi_{1}$. The parity bits RSC 1 and RSC2 are appropriately punctured and multiplexed with the data sequence for the sake of achieving a code rate of 8/9. In order to achieve this, the parity bit sequence of each RSC encoder has 15 punctured bits for every 16 bits. The multiplexed sequence is interleaved by the channel interleaver $\pi_{2}$. Then the interleaved encoded sequence $\underline{x}_{1}^{N}$ is further encoded by a precoder. Finally, the precoded sequence $\underline{a}_{1}^{N}$ is transmitted through the magnetic recording channel.

\section{EQUALIZED LORENTZIAN CHANNEL MODEL}

In saturation-based magnetic recording systems [1], the binary data is recorded along parallel longitudinal tracks of the tape medium by appropriately changing the magnetization of the medium from one direction to the opposite direction in order to represent a bit and vice versa. For example, two of the most common methods of magnetic recording are the so-called NRZ (non-return-to-zero) and NRZI (non-return-to-zero-inverse) methods. In NRZ recording, one direction of magnetization corresponds to a bit " 1 ", while the other corresponds to a bit " 0 ". By contrast, in NRZI recoding, a change in the direction of magnetization corresponds to a bit " 1 ", while no change corresponds to a bit "O". In [2], the NRZI method was shown to be equivalent to the precoding of the data using an encoder polynomial of the form $1 /(1 \oplus D)$ and recording by the NRZ method. This idea allows us to invoke various recording methods by selecting an appropriate precoder polynomial. More specifically, an effective recording method designed for PRML at high recording densities, namely the interleaved NRZI technique was proposed by choosing a precoder polynomial of the form $1 /\left(1 \oplus D^{2}\right)$ in [2]. When the recorded data sequence is read back through the reading head, the playback response of the reading head to the step transition of the magnetization at time 0 can be modeled as a Lorentzian pulse [1]

$$
s(t) \quad=\frac{1}{1+\left(2 t / P W_{50}\right)^{2}},
$$

where $P W_{50}$ is the pulse width at $50 \%$ of its peak value. The resultant continuous-time read-back waveform is assumed to be [24], [25]

$$
r(t) \quad=\sum_{k} a_{k} h(t-k T)+n(t)
$$

where $T$ is the bit duration, the ratio $P W_{50} / T$ is referred to as the recording density, $a_{k} \in\{1,-1\}$ is the data input of the channel, which is usually precoded, and $h(t)$ is the pulse response (sometimes referred to as the dibit response) [25] given by

$$
h(t) \quad=\frac{1}{2}(s(t)-s(t-T)) .
$$

The signal $r(t)$ is passed through a matched filter $h(-t)$. Then the output of the matched filter is sampled at a rate of $1 / T$ and the resultant sampled sequence is given by

$$
u(D) \quad=X_{h}(D) a(D)+v(D),
$$

where $D$ is the unit delay. Furthermore, $X_{h}(D)$ is the autocorrelation sequence of the dibit response, where the $k$-th coefficient is given by

$$
x_{h, k} \quad=\int_{-\infty}^{\infty} h(t) h(t+k T) d t
$$

and $v(D)$ is a Gaussian noise sequence having an autocorrelation sequence of

$$
R_{v}(D) \quad=E\left\{v(D) v\left(D^{-1}\right)\right\}=\left(N_{0} / 2\right) X_{h}(D),
$$

while $N_{0}$ is the single-sided noise power spectral density of the Additive White Gaussian Noise (AWGN) $n(t)$. The matchedfiltered and sampled sequence $u(D)$ is equalized by a finite impulse response (FIR) equalizer $C_{F I R}(D)$ of odd length $N_{E Q}$ [24] according to

$$
C_{F I R}(D)=C_{W}(D) g_{P R}(D),
$$

where $g_{P R}(D)$ is the target response polynomial, while $C_{W}(D)$ is the full response Wiener filter having coefficients given by

$$
\mathbf{c}_{W} \quad=\left(R_{X X}+R_{v}\right)^{-1} \mathbf{X}_{h},
$$

where $R_{X X}$ is an $\left(N_{E Q} \times N_{E Q}\right)$-dimensional correlation matrix, whose $(i, j)$-th element is given by the $(i-j)$-th coefficient of $X_{h}(D) X_{h}\left(D^{-1}\right)$. Furthermore, $R_{v}$ is an $\left(N_{E Q} \times N_{E Q}\right)$-dimensional correlation matrix of the colored noise process $v(D)$, whose $(i, j)$ th element is given by $R_{v,(i-j)}$ and $\mathbf{x}_{h}$ is an $\left(N_{E Q} \times 1\right)$-dimensional vector, whose elements are the coefficients of $X_{h}(D)$ centered at the middle element of $\mathbf{X}_{h}$. When the noise $v(D)$ passes through the equalizer, the equalized noise sequence of

$$
\eta(D) \quad=C_{F I R}(D) v(D)
$$

has the autocorrelation sequence of

$$
R_{\eta}(D) \quad=C_{F I R}(D) C_{F R R}\left(D^{-1}\right) R_{v}(D) .
$$




\section{DECODING SYSTEM}

The decoding system shown in Fig. 2 comprises an outer turbo decoder constituted by a pair of APP decoders and an inner APP detector acting as the equalizer connected through a MUX/puncturing block, a DEMUX/depuncturing block, a channel interleaver $\pi_{2}$ and a channel deinterleaver $\pi_{2}^{-1}$. The turbo decoder of Fig. 2 is matched to the outer turbo encoder, whereas the APP detector is matched to the inner encoder of Fig. 1, which is constituted by the convolution of the target response polynomial $g_{P R}(D)$ and the precoder. The information conveyed by the encoded bits is processed so that only the extrinsic information [12]-[13] is exchanged between the turbo decoder and the APP detector.

\section{A. APP Detector}

The APP detector of Fig. 2 receives both the equalized signals $z_{k}$ from the target response equalizer of Fig. 1 and the $a$ priori information $\operatorname{Pr}\left\{x_{k}\right\}$ of all turbo coded bits $x_{k}$ from the turbo decoder. Then it calculates the extrinsic information $\Gamma_{k}\left(x_{k}\right)$ of the turbo coded bits using the BCJR algorithm. This information will be fed back to the turbo decoder. The trellis diagram of the APP detector is constructed from the combination of the precoder and the target response polynomial. This combination will be referred to as the inner encoder, which can be represented by a recursive convolutional encoder having the feed-forward polynomial $g_{P R}(D)$ and the feed-back polynomial $b(D):=1 \oplus b_{1} D \oplus \ldots \oplus$

$b_{N} D^{N}, b_{i} \in\{0,1\}$, where $1 / b(D)$ represents the precoder function and the notation $\oplus$ stands for modulo-2 addition. Note that the feed-forward polynomial requires arithmetic operations carried out using real numbers whereas the feed-back polynomial requires modulo-2 operations. Since we do not want to increase the number of inner encoder trellis states beyond the minimum required value, the maximum order of $b(D)$ is limited to the order of the target polynomial $g_{P R}(D)$. As a result, the number of the inner encoder's states $S_{k}$ is determined by the order of the target polynomial $g_{P R}(D)$. When the AWGN noise $n(t)$ superimposed at the channel's output passes through the concatenated matched filter, the sampling circuit, and finally the target response equalizer, the resultant discrete noise $\eta(D)$ at the output of the equalizer becomes colored. The channel output response $h(t)$ and the spectral-shaping function of the equalizer $C_{F I R}(D)$ determines the noise correlation function of $(10)$, which is used to calculate the noise prediction/estimation filter coefficients. Again, in this paper we will present three different approaches, which exploit the noise correlation in the APP detector in different ways. Since the actual noise samples are not known in advance, the first approach increases the number of the APP detector's trellis states in order to consider all possible state-transitions affected by the $L$ previous noise samples used in the linear prediction of order $L$. In the second scheme, we will modify the first approach by employing both previous and future noise samples for calculating the current noise estimates. For the sake of implementing this idea, the BCJR of the first approach has to be modified to additionally invoke these future noise samples. According to the third approach, which aims for reducing the complexity of the first approach, the APP detector is designed to cooperate with the VA based linear predictor [9] for ensuring that the number of trellis states is not increased, despite using past noise samples. More specifically, the task of the VA is to find the encoded sequences associated with the survivors of the APP detector's trellis for each iteration of the iterative detection/decoding scheme. Then these sequences are used for the noise prediction embedded in the BCJR algorithm of the APP detector.

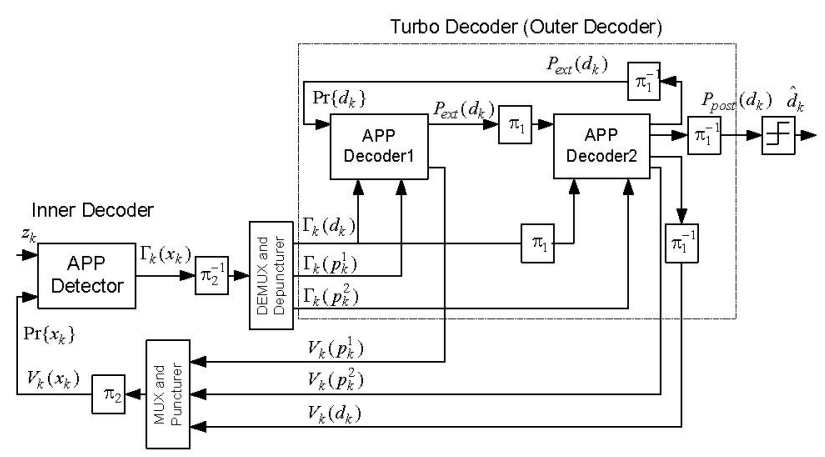

Fig. 2. Concatenated decoder

\section{A.1 APP Detector Using Linear Prediction and an Increased Number of Trellis States (Approach 1)}

According to this approach, the BCJR algorithm is revisited, so that the noise correlation can be exploited with the aid of linear prediction, using previous noise samples only. From our analysis, the forward and backward probabilities can be formulated as follows [23].

$$
\begin{gathered}
\alpha_{k}\left(S_{k-L}, \underline{x}_{k-L+1}^{k}\right) \quad=\alpha\left(S_{k-L}, x_{k-L+1}, \ldots, x_{k}\right) \\
=\sum_{S_{k-L-1}} \sum_{x_{k-L}} M_{k}\left(S_{k-L-1}, \underline{x}_{k-L}^{k}\right) \alpha_{k-1}\left(S_{k-L-1}, \underline{x}_{k-L}^{k-1}\right) \\
\operatorname{Pr}\left\{x_{k}\right\} \operatorname{Pr}\left\{S_{k-L} \mid S_{k-L-1}, x_{k-L}\right\}
\end{gathered}
$$

and

$$
\begin{gathered}
\beta_{k}\left(S_{k-L}, \underline{x}_{k-L+1}^{k}\right)=\beta\left(S_{k-L}, x_{k-L+1}, \ldots, x_{k}\right) \\
=\sum_{S_{k-L+1}} \sum_{x_{k+1}} M_{k+1}\left(S_{k-L}, \underline{x}_{k-L+1}^{k+1}\right) \beta_{k+1}\left(S_{k-L+1}, \underline{x}_{k-L+2}^{k+1}\right) \\
\operatorname{Pr}\left\{x_{k+1}\right\} \operatorname{Pr}\left\{S_{k-L+1} \mid S_{k-L}, x_{k-L+1}\right\},
\end{gathered}
$$

where $L$ is the forward prediction order. The variable $\operatorname{Pr}\left\{x_{k}\right\}$ represents the a priori probability of the turbo encoded bits $x_{k}$, which is supplied by using the corresponding extrinsic information from the turbo decoder, while $\operatorname{Pr}\left\{S_{k} \mid S_{k-1}, x_{k}\right\}$ is the state transition probability of the inner encoder, associated with $x_{k}$,

$$
\operatorname{Pr}\left\{S_{k} \mid S_{k-1}, x_{k}\right\}= \begin{cases}1, & \text { legitimate state transition } \\ 0, & \text { otherwise. }\end{cases}
$$

The tuple $\left(S_{k-L}, \underline{x}_{k-L+1}^{k}\right):=\left(S_{k-L}, x_{k-L+1}, \ldots, x_{k}\right)$ of the inner encoder state $S_{k-L}$ and the subsequent input bits of length $L$ in the lefthand side of (11) may be viewed as the extended states of the APP detector at time $k$. We use $\left(S_{k-L}, \underline{x}_{k-L+1}^{k}\right)$ instead of $\left(S_{k}, \underline{x}_{k-L+1}^{k}\right)$ in order to represent the extended states at time $k$, because the associated paths are uniquely known, regardless of which precoder polynomial is used. For example, when the PR4-equalized channel uses no precoder, each inner encoder state $S_{k}$ can be entered from different states $S_{k-1}$, when encountering the same input $x_{k}$. If the notation $\left(S_{k}, \underline{x}_{k-L+1}^{k}\right)$ is used instead, there are more than one associated paths. As a result, the corresponding inner encoder output sequence $\underline{y}_{k-L}^{k}$ is not exactly known. Hence, the forward/backward recursions cannot be performed with this state definition.

When $\left(S_{k-L-1}, \underline{x}_{k-L}^{k}\right)$ of $M_{k}(\cdot)$ in (11) is given, the noiseless output sequence $\underline{y}_{k-L}^{k}$ of the inner encoder can be exactly determined by encoding the sequence $\underline{x}_{k-L}^{k}:=\left(x_{k-L}, \ldots, x_{k}\right)$ through the inner encoder (with the initial state $S_{k-L-1}$ ). Then, the metric $M_{k}\left(S_{k-L-1}, x_{k-L}^{k}\right)$ based on linear prediction, can be calculated by using the following expression: 


$$
\begin{aligned}
M_{k}\left(S_{k-L-1}, \underline{x}_{k-L}^{k}\right) & =M_{k}\left(\underline{y}_{k-L}^{k}\right) \\
= & \frac{1}{\sqrt{2 \pi \sigma_{p r e d i c t}^{2}}} \exp \left(-\frac{\left(\left(z_{k}-y_{k}\right)-\sum_{i=1}^{L} P_{i}\left(z_{k-i}-y_{k-i}\right)\right)^{2}}{2 \sigma_{\text {predict }}^{2}}\right),
\end{aligned}
$$

where $z_{k}$ is the noise-contaminated signal at the output of the target response equalizer. Furthermore, the variable $\left(z_{k}-y_{k}\right)$ represented the noise process $\eta(D)$ of $(9)$ plus the so-called residue error due to imperfect equalization. The notation $P_{i}$ represents the $i$-th noise prediction coefficient, which can be calculated by using the correlation of the equalized noise samples formulated in (10) of Section III, and $\sigma_{\text {predict }}^{2}$ is the minimum mean-squared noise prediction error. Notice that, from the expressions of the forward and backward probabilities in (11) and (12), respectively, we have to generate the metrics for all possible inner encoder states $S_{k-L-1}$ and for all subsequent input sequences $x_{k-L}^{k}$ of all time instants $k$. Following the evaluation of (11) and (12), the extrinsic information of each encoded bit is calculated as

$$
\begin{array}{r}
\Gamma_{k}\left(x_{k}\right)=\frac{\sum_{S_{k-L-1}^{k-1}} \sum_{x_{k-L}^{k-1}} \alpha_{k-1}\left(S_{k-L-1}, \underline{x}_{k-L}^{k-1}\right) M_{k}\left(S_{k-L-1}, \underline{x}_{k-L}^{k}\right)}{\sum_{x_{k}} \sum_{S_{k-L-1}^{k-L}} \sum_{\underline{x}_{k-L}^{k-1}} \alpha_{k-1}\left(S_{k-L-1}, \underline{x}_{k-L}^{k-1}\right) M_{k}\left(S_{k-L-1}, \underline{x}_{k-L}^{k}\right)} \\
\cdot \frac{\beta_{k}\left(S_{k-L}, \underline{x}_{k-L+1}^{k}\right) \operatorname{Pr}\left\{S_{k-L} \mid S_{k-L-1}, x_{k-L}\right\}}{\beta_{k}\left(S_{k-L}, \underline{x}_{k-L+1}^{k}\right) \operatorname{Pr}\left\{S_{k-L} \mid S_{k-L-1}, x_{k-L}\right\}} .
\end{array}
$$

The denominator in the right-hand side of (15) represents a normalization factor, which renders the summation of $\Gamma_{k}\left(x_{k}\right)$ over all possible $x_{k}$ unity.

\section{A.2 APP Detector Using Linear Estimation and an Increased Number of Trellis States (Approach 2)}

In order to exploit now both the previous and future noise samples for estimating the current one, we define the extended state at time $k$ as follows:

$$
\Delta_{k}=\left(S_{k-L}, x_{k-L+1}^{k+K}\right),
$$

where $L$ and $K$ are the forward and backward prediction orders of the linear estimation, respectively. The metric of the noise estimator used in the APP detector is defined as

$$
\begin{aligned}
M_{k}\left(S_{k-L-1}, \underline{x}_{k-L}^{k+K}\right) & =M_{k}\left(y_{-k-L}^{k+K}\right) \\
= & \frac{1}{\sqrt{2 \pi \sigma_{\text {estimate }}^{2}}} \exp \left(-\frac{\left(\left(z_{k}-y_{k}\right)-\sum_{i=-K, i \neq 0}^{L} P_{i}\left(z_{k-i}-y_{k-i}\right)\right)^{2}}{2 \sigma_{\text {estimate }}^{2}}\right),
\end{aligned}
$$

where $P_{i}$ represents the linear estimator's coefficients, which can be calculated by solving the following set of linear Wiener-Hopf equations:

$$
\left[\begin{array}{cccccc}
R_{\eta, 0} & \cdots & R_{\eta, K-1} & R_{\eta, K+1} & \cdots & R_{\eta, K+L} \\
\vdots & \ddots & \vdots & \vdots & \ddots & \vdots \\
R_{\eta, K-1} & \cdots & R_{\eta, 0} & R_{\eta, 2} & \cdots & R_{\eta, L+1} \\
R_{\eta, K+1} & \cdots & R_{\eta, 2} & R_{\eta, 0} & \cdots & R_{\eta, L-1} \\
\vdots & \ddots & \vdots & \vdots & \ddots & \vdots \\
R_{\eta, K+L} & \cdots & R_{\eta, L+1} & R_{\eta, L-1} & \cdots & R_{\eta, 0}
\end{array}\right]\left[\begin{array}{c}
P_{-K} \\
\vdots \\
P_{-1} \\
P_{1} \\
\vdots \\
P_{L}
\end{array}\right]=\left[\begin{array}{c}
R_{\eta, K} \\
\vdots \\
R_{\eta, 1} \\
R_{\eta, 1} \\
\vdots \\
R_{\eta, L}
\end{array}\right]
$$

and $\sigma_{\text {estimate }}^{2}$ is the minimum mean-squared noise estimation error, given by

$$
\sigma_{\text {estimate }}^{2}=R_{\eta, 0}-\sum_{i=-K, i \neq 0}^{L} P_{i} R_{\eta, i} .
$$

It should be noted that $R_{\eta, i}=R_{\eta,-i}$. To utilize the metric of (17) for the sake of improving the achievable decoding/detection performance, we modified the forward/backward recursions of (11) and (12) in Section A. 1 as follows:

$$
\begin{aligned}
& \alpha_{k}\left(S_{k-L}, \underline{x}_{k-L+1}^{k+K}\right)=\alpha_{k}\left(\Delta_{k}\right) \\
& =\sum_{S_{k-L-1} x_{k-L} M_{k}\left(S_{k-L-1}, x_{k-L}^{k+K}\right) \alpha_{k-1}\left(S_{k-L-1}, \underline{x}_{k-L}^{k+K-1}\right)} \\
& \operatorname{Pr}\left\{x_{k}\right\} \operatorname{Pr}\left\{S_{k-L} \mid S_{k-L-1}, x_{k-L}\right\}
\end{aligned}
$$

and

$$
\begin{array}{r}
\beta_{k}\left(S_{k-L}, \underline{x}_{k-L+1}^{k+K}\right) \quad=\beta_{k}\left(\Delta_{k}\right) \\
=\sum_{S_{k-L+1}} \sum_{k+K+1} M_{k+1}\left(S_{k-L}, \underline{x}_{k-L+1}^{k+K+1}\right) \beta_{k+1}\left(S_{k-L+1}, \underline{x}_{k-L+2}^{k+K+1}\right) \\
\operatorname{Pr}\left\{x_{k+1}\right\} \operatorname{Pr}\left\{S_{k-L+1} \mid S_{k-L}, x_{k-L+1}\right\} \text {. }
\end{array}
$$

By observing, the extended state defined in (16), one might think that it should be identical to the extended state of Approach 1 at time instant $(k+K)$. However, the forward and backward recursions in (20) and (21) handle the extended states differently. Notice that the forward recursion in (21) uses the a priori information $\operatorname{Pr}\left\{x_{k}\right\}$ at time $k$, not that at time $(k+K)$. The extrinsic information of the inner encoder's input is calculated by using

$$
\begin{aligned}
& \Gamma_{k}\left(x_{k}\right)=\frac{\sum_{S_{k-L-1}^{k-L}} \sum_{x_{k-L}^{k-1}} \sum_{x_{k+1}^{k+K}} \alpha_{k-1}\left(\Delta_{k-1}\right) M_{k}\left(S_{k-L-1}, \underline{x}_{k-L}^{k+K}\right)}{\sum_{x_{k}} \sum_{\underline{S}_{k-L-1}^{k}} \sum_{\substack{k+1 \\
k-L}} \sum_{x_{k+1}^{k+K}} \alpha_{k-1}\left(\Delta_{k-1}\right) M_{k}\left(S_{k-L-1}, \underline{x}_{k-L}^{k+K}\right)} \\
& \cdot \frac{\beta_{k}\left(\Delta_{k}\right) \operatorname{Pr}\left\{S_{k-L} \mid S_{k-L-1}, x_{k-L}\right\}}{\beta_{k}\left(\Delta_{k}\right) \operatorname{Pr}\left\{S_{k-L} \mid S_{k-L-1}, x_{k-L}\right\}}
\end{aligned}
$$

For the case of $K=0$, the backward prediction of the estimator is removed. This leads to the first approach outlined in Subsection A.1. On the other hand, if we have $L=0$, the forward prediction disappears, and there is only the backward prediction employed in the APP detector. We have investigated these two special cases and found that their BER performance is identical to each other, provided that the forward prediction order of the first one equals the backward prediction order of the latter one. Moreover, we found that the decoding performance of Approach 2 does not change, if the values of the forward and backward prediction orders are swapped with each other. This is because the noise correlation function is symmetric.

\section{A.3 APP Detector Using Linear Prediction and the Viterbi Algorithm (VA) (Approach 3)}

According to our third approach, the VA is modified to process the a priori information $\operatorname{Pr}\left\{x_{k}\right\}$ of the encoded bits evaluated by the turbo decoder. As we will show, this is achieved without increasing the number of trellis states. In each iteration of the decoding/detection scheme of Fig. 2, the path metric $A_{k}\left(S_{k}\right)$ of the survivor associated with each trellis state $S_{k}$, is recursively calculated as follows:

$$
A_{k}\left(S_{k}\right)=\max _{S_{k-1}}\left(A_{k-1}\left(S_{k-1}\right)+\log \operatorname{Pr}\left\{x_{k}\right\}+\log M_{k}\left(y_{k}, \underline{\hat{y}}_{k-L}^{k-1}\left(S_{k-1}\right)\right)\right),
$$

where $y_{k}$ is the output of the branch $\left(S_{k-1}, S_{k}\right)$, while the variable $\underline{\hat{y}}_{k-L}^{k-1}\left(S_{k-1}\right)$ represents the output sequence of the inner encoder of the survivor associated with state $S_{k-1}$. Let the variable $\hat{S}_{k-1}\left(S_{k}\right)$ denote the inner encoder's state $S_{k-1}$ that satisfies the maximization of (23). Then $\underline{\hat{y}}_{k-L}^{k-1}\left(S_{k-1}\right)$ is the inner encoder's 
output sequence corresponding to the path defined by the following sequence of the inner encoder's states

$$
\left(\hat{S}_{k-L-1}\left(\hat{S}_{k-L}\left(\ldots\left(\hat{S}_{k-2}\left(S_{k-1}\right)\right) \ldots\right)\right), \ldots, \hat{S}_{k-2}\left(S_{k-1}\right), S_{k-1}\right) \text {. }
$$

For the sake of compact notation, we would like to omit the explicit dependence on $S_{k-1}$ and use only $\underline{\hat{y}}_{k-L}^{k-1}$. After the VA has been invoked, the output sequence $\underline{\hat{y}}_{k-L+1}^{k}$ of length $L$ corresponding to the survivor associated with each trellis state $S_{k}$ at all time instant $k$ becomes known. Each sequence is retained and used for the forward/backward recursion as follows:

$$
\alpha_{k}\left(S_{k}\right)=\sum_{S_{k-1}} \sum_{x_{k}} M_{k}\left(y_{k}, \underline{\hat{y}}_{k-L}^{k-1}\right) \alpha_{k-1}\left(S_{k-1}\right) \operatorname{Pr}\left\{x_{k}\right\} \operatorname{Pr}\left\{S_{k} \mid S_{k-1}, x_{k}\right\}
$$

and

$$
\begin{array}{r}
\beta_{k}\left(S_{k}\right)=\sum_{S_{k+1}} \sum_{x_{k+1}} M_{k+1}\left(y_{k+1}, \underline{\hat{y}}_{k-L+1}^{k}\right) \beta_{k+1}\left(S_{k+1}\right) \\
\operatorname{Pr}\left\{x_{k+1}\right\} \operatorname{Pr}\left\{S_{k+1} \mid S_{k}, x_{k+1}\right\},
\end{array}
$$

where $y_{k+1}$ is the output of branch $\left(S_{k}, S_{k+1}\right)$, and $\underline{\hat{y}}_{k-L+1}^{k}$ is the output sequence of the survivor associated with state $S_{k}$. The extrinsic information of each coded bit can be calculated as follows:

$$
\Gamma_{k}\left(x_{k}\right)=\frac{\sum_{\underline{S}_{k-1}^{k}} \alpha_{k-1}\left(S_{k-1}\right) M_{k}\left(y_{k}, \underline{\hat{y}}_{k-L}^{k-1}\right) \beta_{k}\left(S_{k}\right) \operatorname{Pr}\left\{S_{k} \mid S_{k-1}, x_{k}\right\}}{\sum_{x_{k}} \sum_{\underline{S}_{k-1}^{k}} \alpha_{k-1}\left(S_{k-1}\right) M_{k}\left(y_{k}, \underline{\hat{y}}_{k-L}^{k-1}\right) \beta_{k}\left(S_{k}\right) \operatorname{Pr}\left\{S_{k} \mid S_{k-1}, x_{k}\right\}} .
$$

\section{B. Turbo Decoder}

The turbo decoder of Fig. 2 comprises two constituent APP decoders. Each is matched to one of the two RSCs of Fig. 1. The constituent decoders receive the extrinsic information of both the data bits $\Gamma_{k}\left(d_{k}\right)$ and parity bits $\Gamma_{k}\left(p_{k}\right)$ from the APP detector. If the parity bit is punctured, we use $\Gamma_{k}\left(p_{k}=0\right)=\Gamma_{k}\left(p_{k}=1\right)=0.5$. Then the constituent decoders calculate the extrinsic information of both the data bits $V_{k}\left(d_{k}\right)$ and parity bits $V_{k}\left(p_{k}\right)$. Subsequently, the information $V_{k}\left(d_{k}\right)$ and $V_{k}\left(p_{k}\right)$ is appropriately punctured, multiplexed, and used as the a priori probabilities $\operatorname{Pr}\left\{x_{k}\right\}$ of the encoded bits for the APP detector. The extrinsic information $P_{\text {ext }}\left(d_{k}\right)$ of the original uncoded data bits is also calculated and exchanged between the two constituent decoders. After the iterative decoding/detection is completed, the data is decoded as $d_{k}=1$ if the a posteriori probabilities of data bits satisfy $P_{\text {post }}\left(d_{k}=1\right) \geq 0.5$, otherwise as $d_{k}=0$.

\section{PERFORMANCE RESULTS}

In this section the performance of all three decoding schemes is evaluated using computer simulations and compared to that of the benchmark scheme of [18], which does not employ the knowledge of the noise correlation. The recording densities of 2.25 and 2.86 are used. The performance is characterized in terms of the achievable bit error rate (BER) of the decoded data bits. The target polynomial is the PR4-equalized target response. The precoder used in the simulation is $1 /(1 \oplus D)$ (the NRZI recording method).

The number of equalizer taps $N_{E Q}$ was set to 41 . All schemes presented here use the same outer turbo encoder. The two RSC encoders of the outer turbo encoder are identical, using the forward and backward polynomials of $1 \oplus D \oplus D^{3} \oplus D^{4}$ and $1 \oplus D^{3} \oplus D^{4}$, respectively. Regular puncturing patterns were used for both RSC encoders. More specifically, for every sixteen consecutive parity bits of each RSC encoder, the first fifteen parity bits were discarded while the sixteenth parity bit was retained. The turbointerleaved data block size was 4,096 bits. The number of 4096-bit data blocks used in the simulation was 200,000 . A wide range of various noise prediction orders was investigated. Unless stated otherwise, all performance results shown here were record after the fifteenth iteration.

\section{A. Performance Comparison of Presented Schemes}

Figs. 3 and 4 illustrate the BER performance of the three decoding schemes in comparison to that of the benchmark scheme in [18] and to the Noise Predictive Turbo Systems with Hard Feedback (NPTS/HF) proposed in [15] (here we used the linear prediction order of 3 ), at recording densities of 2.25 and 2.86 , respectively. The recording densities of 2.25 and 2.86 which lie in the typical operational range of the recoding densities used for PR4-equalized Lorentzian channels [8]-[10], [24], [26], are selected for the sake of comparison. As we can see, all three decoding schemes exhibit a significant performance improvement over the benchmark scheme. At a BER of $10^{-5}$, for example, coding gains in the range of 1-1.8 $\mathrm{dB}$ can be attained at a recording density of 2.25 , whereas higher gains of $1.8-3.7 \mathrm{~dB}$ are achieved at the higher recording density of 2.86 . When comparing the best BER curves characterizing these schemes at a lower recording density of 2.25 in Fig. 3, it was found that Approach 1 and Approach 2 exhibited a comparable performance, while Approach 3 offered a lower gain, approximately half of that achieved by the other two schemes. However, at the higher recording density of 2.86 seen in Fig. 4, Approach 2 becomes the most effective decoding scheme, offering a $0.7 \mathrm{~dB}$ and $1.6 \mathrm{~dB}$ better coding gain than Approach 1 and Approach 3, respectively. These results indicate that the extra information regarding the correlation of colored noise arisen during the read-back process and after the equalization can be used for enhancing the turbo decoder's performance, if properly exploited.

\section{B. Performance Convergence Behavior}

In this section, we address the performance convergence behavior of the benchmark and the three proposed decoders. First we consider the BER performance achieved at each iteration to quantify how fast the system performance converges to its best possible BER. Fig. 5 depicts the simulation results of all four decoding schemes at a recording density of 2.86. As we can see, the BER performance curves of the benchmark scheme, of Approach 1 and of Approach 2 exhibit a similar behavior. Explicitly, their performance rapidly improves during the early iterations, event after the fourth iteration the additional improvements become negligible, indicating a rapid convergence. For Approach 3, a slightly different convergence behavior is observed. Reduction of the BER is drastic in the first few iterations, but after approximately the fifteenth iteration, the performance curves begin to merge well closely. We also investigated the convergence behavior of these four decoding schemes using different precoders and found that they obeyed similar trends.

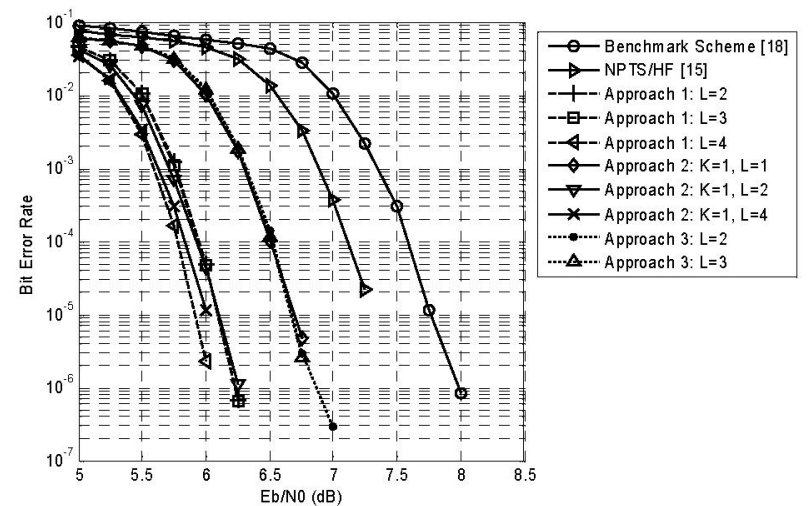

Fig. 3. Performance comparison of various decoding systems at a recording density of 2.25 


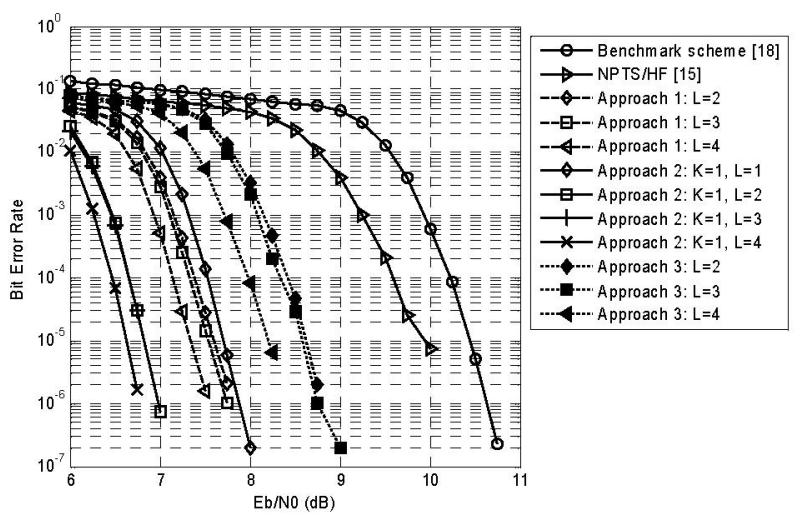

Fig. 4. Performance comparison of various decoding systems at a recording density of 2.86

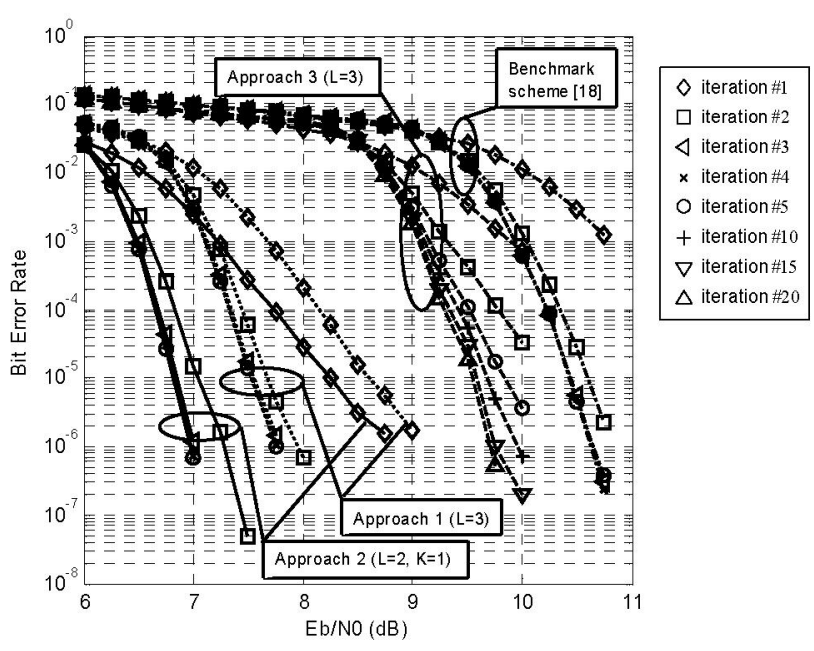

Fig. 5. The performance of various decoding systems at a recording density of 2.86

\section{CONCLUSIONS}

In this paper, three different iterative decoding schemes based on turbo equalization technique with noise prediction/estimation for PR4-equalized magnetic recording Lorentzian channels have been introduced and their performance has extensively been evaluated and compared to a benchmark decoding technique that simply combines turbo coding and turbo equalization without exploiting the noise correlation, for two levels of recording density. As illustrated by simulation results, at a BER of $10^{-5}$, coding gains in the range of $1-1.8 \mathrm{~dB}$ can be attained at a recording density of 2.25 , whereas higher gains of 1.8-3.7 dB are achieved at the higher recording density of 2.86 . This indicates that the three decoding schemes are capable of exploiting the knowledge of noise correlation very effectively, leading to performance improvement of the turbo decoding system in the magnetic recording channels in comparison to that of the benchmark scheme.

\section{REFERENCES}

[1] P. H. Siegel and J. K. Wolf, "Modulation and coding for information storage," IEEE Commun. Magazine, pp. 68-86, Dec. 1991.

[2] H. Kobayashi and D. Tang, "Application of partial response channel coding to magnetic recording systems," IBM J. Res. Dev., pp. 368-375, July 1970 .

[3] P. Kabal and S. Pasupathy, "Partial-response signaling," IEEE Trans. Commun., vol. COM-23, no. 9, pp. 921-934, Sept. 1975.
[4] H. Kobayashi, "Application of Probabilistic Decoding to Digital Magnetic Recording Systems," IBM J. Res. Dev., vol. 15, pp. 6474, Jan. 1971.

[5] R. W. Wood and D. A. Petersen, "Viterbi detection of class IV partial response on a magnetic recording channel," IEEE Trans. Commun., vol. COM-34, no. 5, pp. 454-461, May 1986.

[6] H. K. Thapar and A. M. Patel, "A class of partial response systems for increasing storage density in magnetic recording," IEEE Trans. Magn., vol. MAG-23, no. 5, pp. 3666-3668, Sept. 1987.

[7] W. G. Bliss, "Performance of maximum likelihood detectors in partial erasure," IEEE Trans. Magn., vol. 32, no. 5, pp. 39533955, Sept. 1996.

[8] P. R. Chevillat, E. Eleftheriou, and D. Maiwald, "Noise-predictive partial-response equalizers and applications," in Proc. IEEE Int. Conf. Commun. (ICC'92), vol. 2, pp. 942-947, June 1992.

[9] E. Eleftheriou and W. Hirt, "Improving Performance of PRML/EPRML through noise prediction," IEEE Trans. Magn., vol. 22, no. 5, pp. 3968-3970, Sept. 1996.

[10] J. D. Coker, E. Eleftheriou, R. L. Galbraith, and W. Hirt, "Noisepredictive maximum likelihood (NPML) detection," IEEE Trans. Magn., vol. 34, no. 1, pp. 110-117, Jan. 1998.

[11] R. D. Cideciyan, E. Eleftheriou, and T. Mittelholzer, "Perpendicular and longitudinal recording: a signal-processing and coding perspective," IEEE Trans. Magn., vol. 38, no. 4, pp. 16981704, July 2002.

[12] C. Berrou, A. Glavieux, and P. Thitimajshima, "Near Shannon limit error-correcting coding and decoding: turbo codes," in Proc. IEEE Int. Conf. Comm. (ICC'93), Geneva, Switzerland, May 1993, pp. $1064-1070$.

[13] C. Berrou and A. Glavieux, "Near optimum error correcting coding and decoding: turbo-codes," IEEE Trans. Commun., vol. 44 , no. 10, pp. 1261-1271, Oct. 1996.

[14] T. R. Oenning and J. Moon, "Low density parity check coding for magnetic recording channels with media noise," in Proc. IEEE Int. Conf. Comm. (ICC'01), June 2001, vol. 7, pp. 2189-2193.

[15] Y. Wu and J. R. Cruz, "Noise predictive turbo systems," IEEE Trans. Magn., vol. 37, no. 2, pp. 742-747, Mar. 2001.

[16] Y. Nakamura, Y. Okamoto, H. Osawa, H. Saito, H. Muraoka, and Y. Nakamura, "A Study of turbo decoding with embedded AR channel model for perpendicular recording," IEEE Trans. Magn., vol 39. no. 5, pp. 2570-2572, Sept. 2003.

[17] L. L. McPheters, S. W. McLaughlin, and E. C. Hirsch, "Turbo codes for PR4 and EPR4 magnetic recording." in Proc. The $32^{\text {nd }}$ Asilomar Conf., Nov. 1998, vol. 2, pp. 1778-1782.

[18] T. V. Souvignier, M. Oberg, P. H. Siegel, R. E. Swanson, and J. K. Wolf, "Turbo decoding for partial response channels," IEEE Trans. Commun., vol. 48 , no. 8, pp. 1297-1308, Aug. 2000.

[19] H. Sawaguchi and J. K. Wolf, "Turbo decoding for high-rate concatenated parity-check codes on PRML channels," IEEE Trans. Magn., vol. 36, no. 5, pp. 2173-2175, Sept. 2000.

[20] J. Li, E. Kurtas, K. R. Narayanan, and C. N. Georghiades, "Iterative decoding of turbo product codes over PR-equalized Lorentzian channels with colored noise," in Proc. IEEE Global Telecomm. Conf. (GLOBECOM'01), Nov. 2001, vol. 5, pp. 29722976.

[21] L. R. Bahl, J. Cocke, F. Jelinek, and J. Raviv, "Optimal decoding of linear codes for minimizing symbol error rate," IEEE Trans. Inform. Theory, vol. 20, pp. 284-287, Mar. 1974.

[22] I. D. Marsland and P. T. Mathiopoulos, "Multiple differential detection of parallel concatenated convolutional (turbo) codes in correlated fast Rayleigh fading," IEEE J. Select. Areas Commun., vol. 16, no. 2, pp. 265-275, Feb. 1998.

[23] P. Vanichchanunt, C. Sritiapetch, S. Nakpeerayuth, and L. Wuttisittikulkij, "APP demodulator for turbo coded multiple symbol differential detection under correlated Rayleigh fading channels," in Proc. IEEE Global Telecomm. Conf. (GLOBECOM'04), Dallas, TX, USA, Nov./Dec. 2004, vol. 4, pp.2578-2582.

[24] W. E. Ryan and B. Zafer, "A study of class I partial response signaling for magnetic recording," IEEE Trans. Magn., vol. 33, no. 6, pp. 4543-4550, Nov. 1997.

[25] W. L. Abbott, J. M. Cioffi, and H. K. Thapar, "Performance of digital magnetic recording with equalization and offtrack interference," IEEE Trans. Magn., vol. 27, no. 1, Jan 1991.

[26] R. D. Cideciyan, J. D. Coker, E. Eleftheriou, and R. L. Galbraith, "Noise predictive maximum likelihood detection combined with parity-based post-processing," IEEE Trans. Magn., vol. 37, no. 2, pp.714-720, March 2001. 